\title{
The near-IR surface brightness method applied to six Cepheids in the young LMC cluster NGC 1866
}

\author{
J. Storm ${ }^{1}$, W. P. Gieren ${ }^{2}$, P. Fouqué ${ }^{3}$, T. G. Barnes III ${ }^{4}$, and M. Gómez ${ }^{2}$ \\ 1 Astrophysikalisches Institut Potsdam, An der Sternwarte 16, 14482 Potsdam, Germany \\ e-mail: jstorm@aip.de \\ ${ }^{2}$ Universidad de Concepción, Departamento de Física, Casilla 160-C, Concepción, Chile \\ e-mail: wgieren@coma.cfm.udec.cl; matias@astro-udec.cl \\ 3 Observatoire Midi-Pyrénées, Laboratoire d'Astrophysique (UMR 5572), 14 avenue Édouard Belin, 31400 Toulouse, France \\ e-mail: pfouque@ast.obs-mip.fr \\ 4 The University of Texas at Austin, McDonald Observatory, 1 University Station, C1402, Austin, TX 78712-0259, USA \\ e-mail: tgb@astro.as.utexas.edu
}

Received 15 March 2005 / Accepted 28 May 2005

\begin{abstract}
We present new near-IR light curves for six Cepheids in the young blue LMC cluster NGC 1866 as well as high precision radial velocity curves for ten Cepheids in NGC 1866 and two in NGC 2031. For the six Cepheids in NGC 1866 with new $J$ and $K$ light curves we determine distances and absolute magnitudes by applying the near-IR surface brightness method. We find that the formal error estimates on the derived distances are underestimated by about a factor of two. We find excellent agreement between the absolute magnitudes for the low metallicity LMC Cepheids with the Period-Luminosity (P-L) relation determined by the near-IR surface brightness (ISB) method for Galactic Cepheids suggesting that the slope of the P-L relations for low metallicity and solar metallicity samples could be very similar in contrast to other recent findings. Still there appears to be significant disagreement between the observed slopes of the OGLE based apparent P-L relations in the LMC and the slopes derived from ISB analysis of Galactic Cepheids, and by inference for Magellanic Cloud Cepheids, indicating a possible intrinsic problem with the ISB method itself. Resolving this problem could reaffirm the P-L relation as the prime distance indicator applicable as well to metallicities significantly different from the LMC value.
\end{abstract}

Key words. stars: variables: Cepheids - galaxies: Magellanic Clouds - stars: distances - stars: fundamental parameters galaxies: distances and redshifts

\section{Introduction}

Cepheids still provide one of the most important steps on the extragalactic distance ladder through the Period-Luminosity (P-L) relation. One distinguished example is the major effort to determine the value of the Hubble constant to better than $10 \%$ using the Hubble Space Telescope as presented by Freedman et al. (2001). Still, the zero-point of the P-L relation remains an issue of heated debate and Freedman et al. (2001) chose to adopt a zero-point based on a best estimate of the distance to the Large Magellanic Cloud of $18.50 \pm 0.1 \mathrm{mag}$, and to assume

\footnotetext{
* Based on data acquired at the Las Campanas Observatory, Chile, the Cerro Tololo Inter American Observatory, Chile, and the European Southern Observatory, Chile.

$\star \star$ Full Tables 1, 2 and 4 are available in electronic form at the CDS via anonymous ftp to cdsarc.u-strasbg.fr (130.79.128.5) or via http://cdsweb.u-strasbg.fr/cgi-bin/qcat?]/A+A/440/487

$\star \star \star$ Appendix $\mathrm{A}$ is only available in electronic form at http://www.edpsciences.org
}

a weak, but still ill-defined, effect on the zero-point as a function of metallicity. For a recent summary of the spread of current LMC distance estimates see e.g. Gibson (2000) and Benedict et al. (2002).

Fouqué et al. (2003) and Storm et al. (2004b) (S04 hereinafter) have determined direct distances and absolute magnitudes to a large sample of Galactic Cepheids using the near-IR surface brightness (ISB) method as calibrated by Fouqué \& Gieren (1997). Unfortunately the slopes of the derived Galactic P-L relations differ from those observed in the LMC by OGLE (Udalski et al. 1999) in the optical and by Persson et al. (2004) in the near-IR. If this effect is caused by the difference in metallicity of the two Cepheid populations it will have harmful consequences for the use of the P-L relation as a distance indicator.

Here we present new data for a sample of short period LMC cluster Cepheids and extend the ISB analysis to six Cepheids in the blue populous LMC cluster NGC 1866. This analysis will allow us to compare directly the absolute magnitude of LMC Cepheids with those for Galactic Cepheids using exactly the same procedure for both samples. It will also 
provide a first direct comparison of the slope of the P-L relations for low metallicity stars in the Magellanic Clouds with the P-L relation for near solar metallicity Galactic Cepheids by including the SMC Cepheids studied by S04.

Additionally we will exploit the fact that the NGC 1866 Cepheids can all be considered to be at the same distance so we can measure directly the dispersion of the measured distances and thereby the true random error estimate for the method, something which has previously not been possible.

NGC 1866 is a unique object for many types of studies. It is young ( $\approx 100 \mathrm{Myr}$, Brocato et al. 1989), and it is at the same time metal-poor when compared to similarly young Galactic stars. Hill et al. (2000) find from high resolution spectroscopy of three giants a value of $[\mathrm{Fe} / \mathrm{H}]=-0.5 \pm 0.1$ confirming the photometric measurement of $[\mathrm{Fe} / \mathrm{H}]=-0.46 \pm 0.18$ by Hilker et al. (1995). It is also very populous and thus provides an excellent laboratory for studying stellar evolution for intermediate mass stars (e.g. Brocato et al. 2003 and references therein). In the present context the most notable feature is the large population of cluster Cepheids. The possible association of Cepheids with NGC 1866 was noticed early by Shapley \& Nail (1950) and photographic light curves were published by Arp $\&$ Thackeray (1967) for seven stars, six of which are analyzed in the present paper. Additional Cepheids were identified by Storm et al. (1988), Welch et al. (1991) (W91 hereinafter), Welch \& Stetson (1993) and most recently, using Hubble Space Telescope data, by Brocato et al. (2004). The Cepheids in the most crowded parts of the cluster are presently too crowded to be studied accurately from the ground, still the list of Cepheids presented by Welch \& Stetson (1993) has more than 20 entries of which most have determined periods. NGC 1866 thus provides us with a unique laboratory to study the Cepheid instability strip, which has already been exploited by e.g. W91, Bertelli et al. (1993), and Brocato et al. (2004).

Côté et al. (1991) performed the first Baade-Wesselink type analysis of Cepheids in NGC 1866 based on optical data, and the present analysis will build on their observational data as well as our new data. W91 provide an excellent historical summary of the research on the interesting cluster NGC 1866 up to the time of that paper.

Gieren et al. (2000b) presented a first near-IR surfacebrightness analysis for the NGC 1866 Cepheid HV 12198, and we present here a re-analysis of this star based on the same precepts as employed for the other Cepheids in the Milky Way and in the SMC.

\section{The sample}

For the present work we have obtained $J$ and $K$ light curves as well as radial velocity curves for a sample of Cepheids in the young blue populous clusters NGC 1866 and NGC 2031. Finding charts for the NGC 1866 stars can be found in Storm et al. (1988) (for NGC 1866-V4 see W91 though) and for the NGC 2031 stars in Mateo (1992).

Only the data for the NGC 1866 Cepheids with Harvard Variable (HV) numbers proved of sufficient quality and completeness to warrant the application of the infrared surface brightness method.

\section{The optical photometry}

Optical light curves using CCD detectors have been obtained for a number of NGC 1866 Cepheids by Walker (1987), W91, and most recently by Gieren et al. (2000a). As shown by Gieren et al. (2000a) these data sets are all in very good agreement, especially in the $V$-band, and taken together they provide excellent phase coverage and high accuracy for the six Cepheids which are of main interest to the present paper. Consequently we have adopted this combined $B V R I$ data set for further analysis. To ensure that all the data are on the same system we have applied small offsets (few hundredth of a mag) to the various data sets to bring them into optimal agreement with the Gieren et al. data set.

The ephemerides which we have used are from Gieren et al. (2000a) for the stars with HV numbers, for the remaining stars we have determined periods and epochs on the basis of the available radial velocity data (see also Sect. 5.3) and they are summarized in Table 3. W91 found evidence for a slightly variable period for HV 12198 but we do not find any significant variation over the 11 year period spanned by the optical data from Walker (1987), W91, and Gieren et al. (2000a), so we have simply adopted a constant period for this star.

\section{The near-IR data}

We have obtained near-IR photometry in the $J$ and $K^{\prime}$ bands at ESO, La Silla, using the ESO-MPG $2.2 \mathrm{~m}$ telescope with the IRAC-2 camera during observing runs in Dec. 1996 and Jan. 1998. We have also acquired photometry in the $J$ and $K$ s bands at the Las Campanas Observatory (LCO) using the $1 \mathrm{~m}$ Swope telescope with the IRC in Nov. 1998 and Jan. 1999, and the $2.5 \mathrm{~m}$ du Pont telescope in Jan. 1999. Finally we used the OSIRIS instrument at the $1.5 \mathrm{~m}$ telescope at the Cerro Tololo Inter-American Observatory in Dec. 2000 to obtain $J$ and $K$ s photometry.

\subsection{Data reduction}

For all the cameras we applied the appropriate non-linearity correction as quoted in the manuals. The exposure times were chosen to avoid exposing the detector to more than half of full well, so in all cases the applied non-linearity correction remained small.

The data were obtained in sets where one set typically consisted of five or six different pointings on the target. In this way a local sky frame could be determined from the other frames in a set by median filtering. These local skyframes were subtracted from each of the original frames. In the case of more crowded fields, i.e. close to the cluster center, it was necessary to use sky frames based on data sets from another target with same exposure parameters obtained close in time.

In case of the La Silla data the flat fields were determined by subtracting a domeflat with the quartz lamp off from a dome flat with the quartz lamp on. A number of these exposure sets were averaged together to achieve a very high signal to noise ratio. A set of observations of a standard star placed in a grid of 4 by 4 positions on the chip was used to determine the 
Table 1. The $K$ and $(J-K)$ photometric measurements for the program stars transformed to the CIT system as described in the text. The complete table is available electronically from the CDS.

\begin{tabular}{cccccc}
\hline \hline ID & $\begin{array}{c}\text { HJD } \\
\text { [days] }\end{array}$ & $\begin{array}{c}K \\
{[\mathrm{mag}]}\end{array}$ & $\begin{array}{c}\sigma_{(K)} \\
{[\mathrm{mag}]}\end{array}$ & $\begin{array}{c}(J-K) \\
{[\mathrm{mag}]}\end{array}$ & $\begin{array}{c}\sigma_{(J-K)} \\
{[\mathrm{mag}]}\end{array}$ \\
\hline HV 12197 & 2450438.65133 & 14.524 & 0.039 & 0.481 & 0.045 \\
HV 12197 & 2450439.57052 & 14.428 & 0.026 & 0.327 & 0.028 \\
HV 12197 & 2450439.65912 & 14.387 & 0.024 & 0.354 & 0.026 \\
HV 12197 & 2450439.76887 & 14.399 & 0.018 & 0.362 & 0.020 \\
HV 12197 & 2450439.86449 & 14.408 & 0.030 & 0.365 & 0.032 \\
HV 12197 & 2450440.55941 & 14.382 & 0.027 & 0.417 & 0.030 \\
HV 12197 & 2450440.67597 & 14.387 & 0.022 & 0.427 & 0.027 \\
HV 12197 & 2450440.79185 & 14.402 & 0.017 & 0.458 & 0.028 \\
HV 12197 & 2450441.76019 & 14.610 & 0.015 & 0.403 & 0.032 \\
cont. & & & & & \\
\hline
\end{tabular}

illumination correction to be applied to the flatfield exposures and this corrected flatfield was used to flat field the science exposures.

In the case of the Las Campanas data we used twilight flatfields. Here the signal to noise ratio was somewhat lower, as the exposure level could not be as finely adjusted as was the case for the La Silla data. No additional correction for illumination effects was included.

Finally the bad pixels were eliminated using the IRAF fixpix routine.

\subsection{Photometry}

The PSF-fitting photometry was carried out with DoPHOT2.0 (Schechter et al. 1993) running on a DEC-Alpha with OSF/1 UNIX.

For each field a database was constructed containing the photometry of the stars from all the individual exposures of the field. On the basis of this database comparison stars could be selected and used as a reference to transform the photometry of each exposure to the instrument system. The detailed transformation procedure is described in the Appendix and the calibrated photometric data is tabulated in Table 1 and the resulting light and color curves have been plotted in Figs. 1-6.

\section{The radial velocities}

The radial velocity curves are based on high resolution spectra obtained with the Las Campanas Observatory $2.5 \mathrm{~m} \mathrm{du}$ Pont telescope equipped with the echelle spectrograph and the 2D-Frutti photon counting system. This system provides a dispersion of $0.2 \mathrm{~nm} / \mathrm{mm}\left(\approx 0.006 \mathrm{~nm} \mathrm{pix}^{-1}\right)$ and was used with a slit of $1.5 \times 4$ arcsec which transforms into an instrumental profile with a $F W H M$ of approximately 3.5 pixels or $12 \mathrm{~km} \mathrm{~s}^{-1}$ corresponding to a resolving power of $R \approx 20000$.

We also used the Cerro Tololo $4 \mathrm{~m}$ Blanco telescope with the echelle spectrograph, the red long camera and the Tek2048 CCD detector. This system provides a very

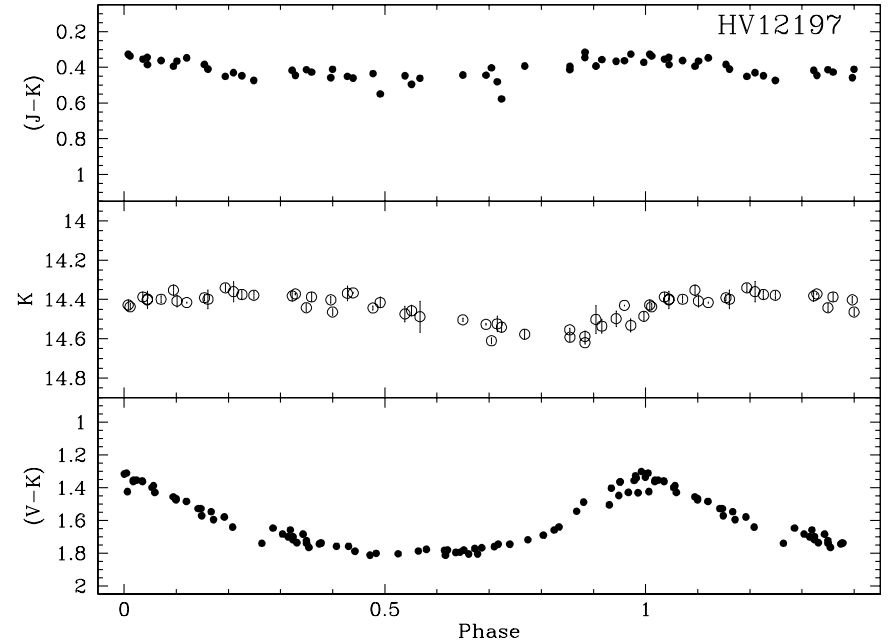

Fig. 1. $K$-band light curve and $(V-K)$ and $(J-K)$ colour curves for HV 12197.

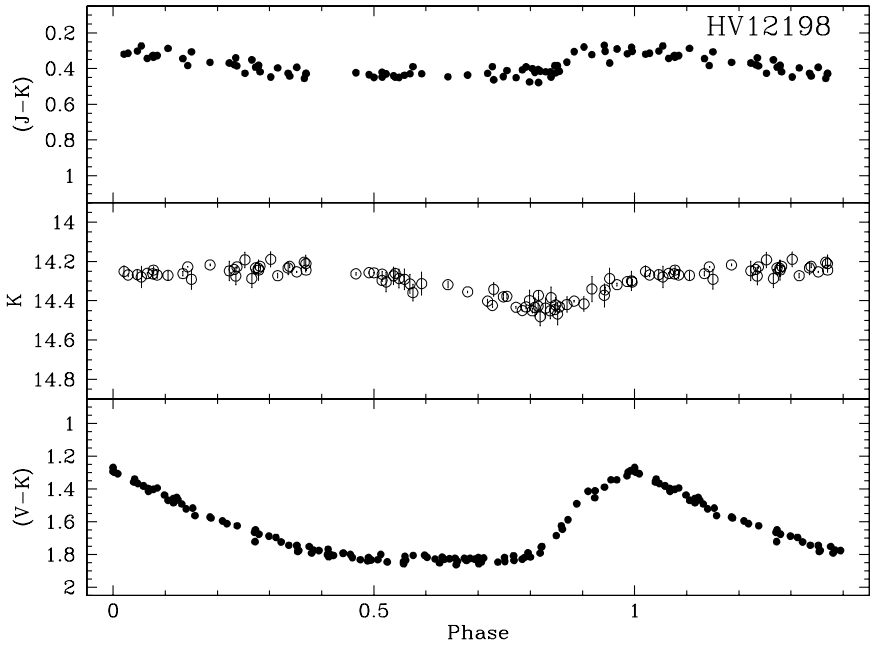

Fig. 2. $K$-band light curve and $(V-K)$ and $(J-K)$ colour curves for HV 12198.

similar resolving power $(R \approx 20000)$ with a dispersion of about $0.25 \mathrm{~nm} / \mathrm{mm}$ which combined with $0.024 \mathrm{~mm}$ pixels again gives about $0.006 \mathrm{~nm} \mathrm{pix}^{-1}$. The slit was again set at $1.5 \mathrm{arcsec}$ width. Integration times for our science objects with both systems were between 20 and 45 min depending on the weather conditions and the brightness of the star.

\subsection{The observations}

The Las Campanas data were obtained during three observing runs in Dec. 1995, Jan. 1996 and Jan. 2000, and the CTIO data were obtained during a single run in Jan. 2000. The corresponding Heliocentric Julian Dates are tabulated in Table 2 with the derived velocities.

The science spectra were interlaced with Th-Ar lamp spectra, and as the science objects were all in the same part of the sky, most science exposures had bracketing calibration spectra. A number of radial velocity standard stars were observed during each run to check the zero point. During the CTIO run, twilight sky exposures were obtained for the same purpose. 


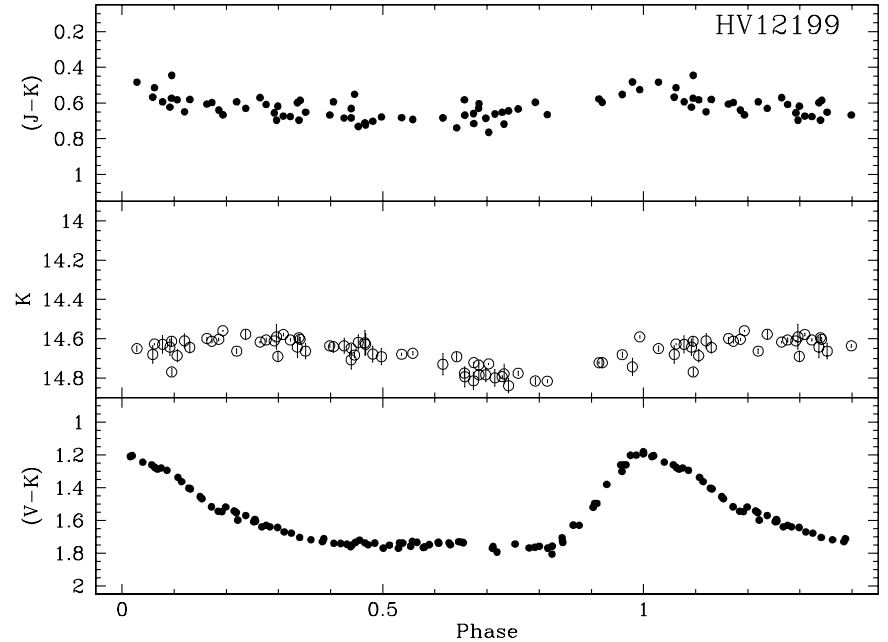

Fig. 3. $K$-band light curve and $(V-K)$ and $(J-K)$ colour curves for HV 12199.

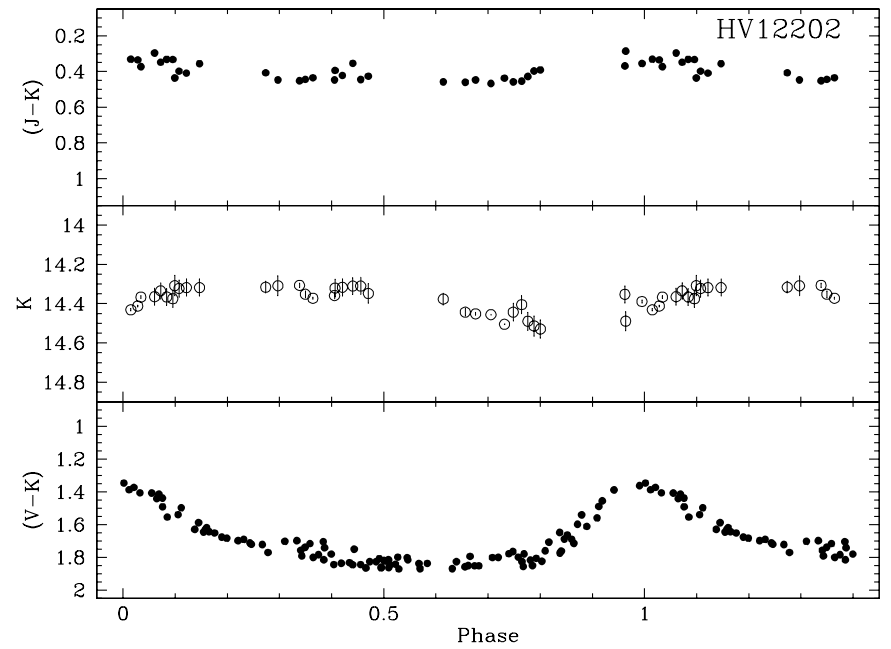

Fig. 4. $K$-band light curve and $(V-K)$ and $(J-K)$ colour curves for HV 12202.

The bright star AT67A (Arp \& Thackeray 1967), earlier selected by W91 to be similar to the Cepheids in spectral type and with sharp lines, which is located close to the two stellar clusters was observed frequently and served as our crosscorrelation template star.

\subsection{Data reduction}

The Las Campanas data were first divided by a lamp flat field to take out the pixel to pixel variation in the detector. The apertures corresponding to each echelle order were extracted using the IRAF $^{1}$ noao.imred.echelle. apall command where the individual apertures had first been traced on a well exposed stack of standard star frames. The calibration Th-Ar apertures were extracted for each science exposure using

${ }^{1}$ IRAF is the Image Reduction and Analysis Facility, made available to the astronomical community by the National Optical Astronomy Observatories, which are operated by AURA, Inc., under cooperative agreement with the National Science Foundation.

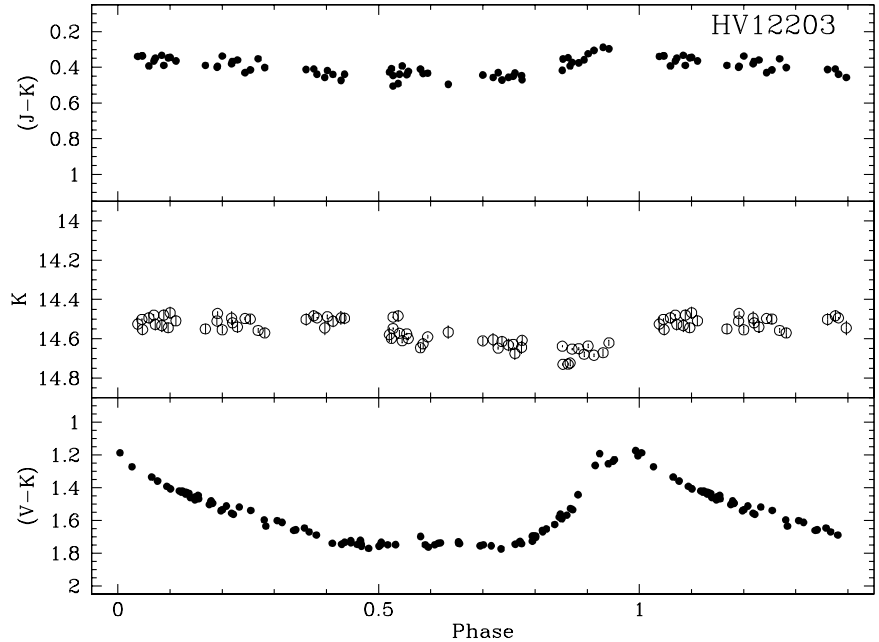

Fig. 5. $K$-band light curve and $(V-K)$ and $(J-K)$ colour curves for HV 12203.

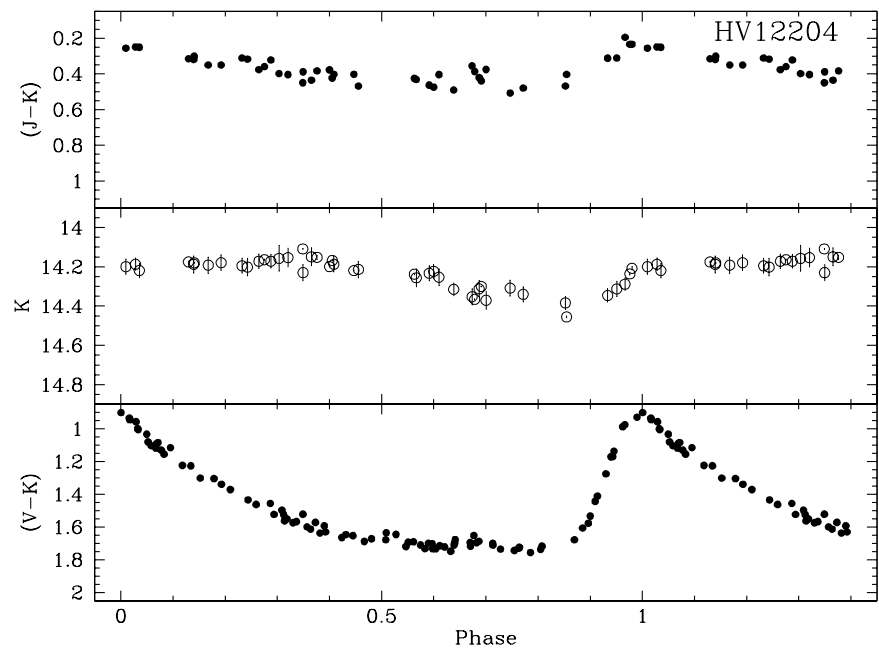

Fig. 6. $K$-band light curve and $(V-K)$ and $(J-K)$ colour curves for HV 12204.

the apertures previously defined by the corresponding science exposure.

The wavelength solution was determined for each calibration Th-Ar spectrum and using the IRAF refspectra and dispcor commands the science spectra were wavelength calibrated by weighting the bracketing Th-Ar spectra according to their closeness in time to the science exposure.

Ten echelle orders covering the wavelength range from 444-513 nm were extracted and continuum corrected before they were merged into a one-dimensional spectrum using the scombine command. Finally outlying points, mostly from the low signal-to-noise ends of the spectral orders were removed to eliminate spurious signals.

The CTIO CCD data were bias subtracted with the bias level corresponding to each individual quadrant of the quadread CCD and each quadrant had its gain normalized. No pixel to pixel variation correction (flatfielding) was deemed necessary, and we preferred to refrain from such a correction to avoid the introduction of additional noise into the data. For the longer 
Table 2. The barycentric radial velocity measurements, $V_{\mathrm{r}}$, for each star, with the associated Heliocentric Julian Date, HJD, and phase, $\phi$. The estimated random error for all the velocities is of the order $1 \mathrm{~km} \mathrm{~s}^{-1}$. The complete table is available from the CDS.

\begin{tabular}{|c|c|c|c|c|c|c|c|c|c|c|c|}
\hline $\begin{array}{c}\text { HJD } \\
-2400000 \\
\text { [days] }\end{array}$ & $\phi$ & {$\left[\mathrm{km} \mathrm{s}^{-1}\right]$} & $\begin{array}{c}\text { HJD } \\
-2400000 \\
\text { [days] }\end{array}$ & $\phi$ & {$\left[\mathrm{km} \mathrm{s}^{-1}\right]$} & $\begin{array}{c}\text { HJD } \\
-2400000 \\
\text { [days] }\end{array}$ & $\phi$ & {$\left[\mathrm{km} \mathrm{s}^{-1}\right]$} & $\begin{array}{c}\text { HJD } \\
-2400000 \\
\text { [days] }\end{array}$ & $\phi$ & {$\left[\mathrm{km} \mathrm{s}^{-1}\right]$} \\
\hline \multicolumn{3}{|c|}{ HV 12197} & 50097.6485 & 0.70 & 320.1 & \multicolumn{3}{|c|}{ HV 12204} & 50096.5568 & 0.58 & 304.3 \\
\hline 50066.6647 & 0.39 & 298.5 & 51555.7082 & 0.17 & 286.3 & 50066.5654 & 0.15 & 282.2 & 50096.7672 & 0.68 & 306.5 \\
\hline 50067.5543 & 0.67 & 314.2 & 51556.5402 & 0.48 & 308.8 & 50066.7376 & 0.20 & 284.1 & 50097.5561 & 0.07 & 293.2 \\
\hline 50067.8071 & 0.76 & 315.5 & 51556.6603 & 0.53 & 310.1 & 50067.6675 & 0.47 & 302.3 & 50097.7846 & 0.19 & 289.7 \\
\hline 50068.6029 & 0.01 & 283.8 & 51556.8110 & 0.58 & 314.4 & 50068.7943 & 0.80 & 318.9 & & & \\
\hline 50095.7638 & 0.65 & 310.9 & 51557.6894 & 0.92 & 292.6 & 50096.7144 & 0.92 & 304.9 & \multicolumn{3}{|c|}{ NGC 2031-V4 } \\
\hline 50096.6082 & 0.92 & 290.1 & 51558.7151 & 0.31 & 297.4 & 50097.7381 & 0.21 & 286.8 & 51555.5790 & 0.01 & 224.5 \\
\hline 50096.8013 & 0.98 & 281.6 & 51572.7110 & 0.61 & 315.3 & 51555.6398 & 0.18 & 284.3 & 51555.7933 & 0.07 & 228.3 \\
\hline 50097.6318 & 0.24 & 292.6 & 51574.7432 & 0.38 & 300.0 & 51556.6832 & 0.48 & 304.0 & 51556.5662 & 0.29 & 243.2 \\
\hline 50097.8436 & 0.31 & 292.1 & & & & 51558.5679 & 0.03 & 275.1 & 51556.7349 & 0.34 & 245.8 \\
\hline 51555.6825 & 0.03 & 282.5 & \multicolumn{3}{|c|}{ HV 12202} & 51558.6887 & 0.06 & 276.0 & 51557.7196 & 0.63 & 260.9 \\
\hline 51555.8249 & 0.07 & 278.6 & 50066.6821 & 0.43 & 314.6 & 51571.7364 & 0.86 & 323.3 & 51558.5926 & 0.89 & 248.2 \\
\hline 51556.7124 & 0.35 & 298.5 & 50067.6265 & 0.73 & 326.5 & 51573.7296 & 0.44 & 302.0 & 51571.6981 & 0.71 & 267.4 \\
\hline 51556.8360 & 0.39 & 301.0 & 50067.8286 & 0.80 & 325.7 & & & & 51571.8097 & 0.74 & 267.5 \\
\hline 51558.5401 & 0.94 & 287.3 & 50068.7466 & 0.09 & 292.0 & \multicolumn{3}{|c|}{ NGC 1866-V4 } & 51572.5492 & 0.96 & 228.1 \\
\hline 51571.5869 & 0.09 & 282.2 & 50095.8325 & 0.83 & 321.1 & 50067.7223 & 0.77 & 310.5 & 51572.6442 & 0.98 & 227.7 \\
\hline 51572.7538 & 0.46 & 304.1 & 50096.6756 & 0.10 & 292.1 & 50068.6640 & 0.05 & 293.5 & 51572.7860 & 0.03 & 225.8 \\
\hline 51573.5842 & 0.72 & 316.6 & 50097.6901 & 0.43 & 313.2 & 50097.5985 & 0.77 & 312.6 & 51573.5461 & 0.25 & 241.7 \\
\hline 51573.7776 & 0.78 & 315.2 & 51571.6203 & 0.72 & 308.1 & 50097.8082 & 0.83 & 306.7 & 51573.6566 & 0.28 & 244.1 \\
\hline & & & 51572.6177 & 0.04 & 275.1 & & & & 51574.5423 & 0.54 & 259.8 \\
\hline \multicolumn{3}{|c|}{ HV 12198} & 51573.6939 & 0.38 & 293.0 & \multicolumn{3}{|c|}{ NGC 1866-V6 } & 51574.6699 & 0.57 & 262.2 \\
\hline 50066.6203 & 0.16 & 284.0 & 51574.6297 & 0.69 & 307.4 & 50067.7598 & 0.72 & 306.2 & 51574.7803 & 0.61 & 263.2 \\
\hline 50066.7786 & 0.20 & 289.4 & & & & 50068.6848 & 0.20 & 291.2 & & & \\
\hline 50067.6057 & 0.44 & 304.1 & \multicolumn{3}{|c|}{ HV 12203} & 50095.7013 & 0.15 & 293.2 & \multicolumn{3}{|c|}{ NGC 2031-V11 } \\
\hline 50068.7307 & 0.76 & 318.7 & 50066.5904 & 0.27 & 294.1 & 50096.5415 & 0.59 & 309.6 & 51555.5537 & 0.25 & 236.7 \\
\hline 50095.8158 & 0.44 & 307.2 & 50066.7585 & 0.32 & 302.1 & 50096.7436 & 0.69 & 311.4 & 51555.7669 & 0.32 & 243.3 \\
\hline 50096.6464 & 0.68 & 316.2 & 50067.6466 & 0.62 & 315.4 & 50097.5401 & 0.10 & 290.2 & 51556.5884 & 0.61 & 263.9 \\
\hline 50096.8256 & 0.73 & 314.9 & 50067.8480 & 0.69 & 319.6 & 50097.7700 & 0.22 & 295.0 & 51556.7571 & 0.67 & 265.4 \\
\hline 50097.6714 & 0.97 & 281.8 & 50067.8637 & 0.70 & 319.2 & & & & 51558.6141 & 0.33 & 245.0 \\
\hline 51571.5604 & 0.36 & 301.0 & 50068.7692 & 0.00 & 284.8 & \multicolumn{3}{|c|}{ NGC 1866-V7 } & 51571.6610 & 0.95 & 221.2 \\
\hline & & & 50095.8520 & 0.17 & 290.5 & 50066.7189 & 0.65 & 309.6 & 51571.7738 & 0.99 & 213.8 \\
\hline \multicolumn{3}{|c|}{ HV 12199} & 50096.6908 & 0.45 & 308.3 & 50067.6876 & 0.94 & 294.1 & 51572.5861 & 0.28 & 241.3 \\
\hline 50066.6376 & 0.95 & 286.7 & 50097.7193 & 0.80 & 318.3 & 50068.6265 & 0.21 & 295.4 & 51572.8168 & 0.36 & 244.6 \\
\hline 50066.8064 & 0.01 & 278.8 & 51555.6186 & 0.32 & 300.9 & 50096.5853 & 0.47 & 298.2 & 51573.6204 & 0.64 & 263.1 \\
\hline 50067.5748 & 0.30 & 293.4 & 51555.7429 & 0.36 & 303.3 & 50096.7818 & 0.52 & 297.9 & 51574.5781 & 0.98 & 220.5 \\
\hline 50067.7814 & 0.38 & 303.5 & 51556.6311 & 0.66 & 319.4 & 50097.5797 & 0.76 & 310.8 & 51574.7004 & 0.03 & 222.1 \\
\hline 50068.5866 & 0.69 & 319.7 & 51556.7839 & 0.71 & 322.0 & 50097.8276 & 0.83 & 312.2 & 51574.8164 & 0.07 & 223.5 \\
\hline 50068.8237 & 0.78 & 316.9 & 51557.6672 & 0.01 & 283.9 & & & & & & \\
\hline 50095.7790 & 0.99 & 277.6 & 51558.6506 & 0.34 & 301.5 & \multicolumn{3}{|c|}{ NGC 1866-V8 } & & & \\
\hline 50096.6235 & 0.31 & 295.9 & 51572.6850 & 0.10 & 285.6 & 50067.7022 & 0.20 & 286.6 & & & \\
\hline 50096.8422 & 0.39 & 299.4 & & & & 50068.6428 & 0.67 & 311.7 & & & \\
\hline
\end{tabular}

exposures it was necessary to remove the cosmic ray hits, and this was achieved by simply eliminating counts higher than a certain threshold together with the pixels within a 2 pixel radius.
The spectral retrieval proceeded as for the Las Campanas data and 10 echelle orders covering the range 480-529 nm were finally extracted. The length of the extracted onedimensional spectra were in both cases limited by the fact that 
Table 3. The ephemerides for the stars.

\begin{tabular}{rll}
\hline \hline ID & $\begin{array}{l}\text { Period } \\
\text { [days] }\end{array}$ & $\begin{array}{l}\text { Epoch of max. } V \text { light } \\
\text { [HJD] }\end{array}$ \\
\hline HV 12197 & 3.14381 & 2450071.72 \\
HV 12198 & 3.52279 & 2450069.59 \\
HV 12199 & 2.63918 & 2450103.72 \\
HV 12202 & 3.10112 & 2450071.56 \\
HV 12203 & 2.95411 & 2450071.715 \\
HV 12204 & 3.43876 & 2450069.49 \\
NGC 1866-V4 & 3.31895 & 2450068.5 \\
NGC 1866-V6 & 1.93386 & 2450068.3 \\
NGC 1866-V7 & 3.388369 & 2450067.9 \\
NGC 1866-V8 & 2.00714 & 2450067.3 \\
NGC 2031-V4 & 3.42871 & 2451572.7 \\
NGC 2031-V11 & 2.8244 & 2451571.8 \\
\hline
\end{tabular}

the cross-correlation procedure used can only handle spectra with a maximum of 8192 pixels.

The spectra were cross-correlated numerically using the technique developed by Tonry \& Davis (1979) implemented in the rvsao package (Kurtz \& Mink 1998) within IRAF.

For each run the spectra of the local reference star AT67A after dispersion correction were stacked to provide a high signal to noise reference template spectrum. The science spectra were then cross-correlated against this template, for which we adopted a velocity of $-0.7 \mathrm{~km} \mathrm{~s}^{-1}$ as already found by W91 and confirmed by our measurements with respect to a twilight sky template and the standard stars observed.

Nightly radial velocity offsets were determined on the basis of the shifts of the individual AT67A observations and/or twilight sky spectra against the template and these offsets were finally added to the derived velocities. Using the rvsao package the observed radial velocities were transformed into barycentric velocities, which are the values tabulated in Table 2 . The phases listed in the table are based on the ephemerides from Table 3.

\subsection{Radial velocity curves}

The radial velocity curves are shown in Figs. 7-8, where our observations are shown with filled symbols and open symbols are used for data from the literature. We find excellent agreement with the data from W91 and with Storm et al. (2004a) for most of the stars. For HV 12203 it appears that one of the observations from W91 is a misidentification (phase $=0.68$ ) and it has been rejected in the following. Two stars, HV 12202 and HV 12204 show a very systematic shift between observing runs, and we strongly suspect this is due to orbital motion. The case for HV 12202, as earlier noted by W91, is particularly strong as the offset is present in several observing runs. In the case of HV 12204 the shift is only seen for the W91 data. On the other hand we see no reason why the velocities for a single star should be systematically off when the rest of the observations from that run are in good agreement with the other runs. To provide a radial velocity curve which is useful for a Baade-Wesselink analysis we have determined the shifts for HV 12202 to be $+21 \mathrm{~km} \mathrm{~s}^{-1}$ for the W91 data from the period $2447860<\mathrm{HJD}<2447874$ and our observations from the period $2451571<$ HJD $<2451575$ should be shifted by $+18 \mathrm{~km} \mathrm{~s}^{-1}$. In the case of HV 12204 the W91 data from the same period as for HV 12202 should be shifted by $+7 \mathrm{~km} \mathrm{~s}^{-1}$. The resulting radial velocity curves are plotted in Fig. 9.

We have determined the systemic velocity, $V_{\gamma}$, for each star as the phase weighted mean velocity. These values are tabulated in Table 4. For the two stars showing orbital motion, HV 12202 and HV 12204, we have performed the phase weighting on the original, unshifted, data. However, due to the limited sampling of the orbital motion a significant, but unknown, uncertainty remains on the systemic velocity for these stars.

For the two Cepheids in NGC 2031 and for the four NGC 1866 stars, V4, V6, V7, and V8, we have determined rough ephemerides as tabulated in Table 3 on basis of the radial velocity curves. The epochs of maximum light have been estimated by forcing the minimum radial velocity to occur at phase 0.0 , which seems reasonable from the radial velocity curves for the other stars in Figs. 7 and 9.

\section{The infrared surface brightness method}

The surface brightness method was developed in a series of papers by Barnes \& Evans (1976) and Barnes et al. (1976, 1977). They determined a calibration of the colour index $(V-R)$ through a linear relation to the surface brightness parameter $F_{V}$ defined as

$$
F_{V}(\phi)=4.2207-V_{0}-0.5 \log \theta(\phi)
$$

where $\theta$ is the stellar angular diameter, $V_{0}$ the de-reddened visual magnitude, and $\phi$ is the phase.

The angular diameter is geometrically related to the distance and stellar radius through

$\theta(\phi)=2 R(\phi) / d=2\left(R_{0}+\Delta R(\phi)\right) / d$

where $d$ is the distance and $R$ is the radius. The variation of the radius can be determined by integrating the radial velocity curve of the star:

$\Delta R(\phi)=\int-p\left[V_{\mathrm{r}}(\phi)-V_{\gamma}\right] \mathrm{d} \phi$

where $p$ is the so called projection factor converting radial velocity into pulsational velocity, $V_{\mathrm{r}}(\phi)$ is the radial velocity and $V_{\gamma}$ is the systemic velocity.

As $F_{V}$ is calibrated from interferometric measurements of stellar angular diameters it is then possible to solve Eq. (2) for the two remaining unknowns, namely the distance, $d$, and the stellar mean radius, $R_{0}$. As discussed by Barnes et al. (2003) and S04, it is sometimes necessary to apply a small phase shift, $\Delta \phi$, to the radial velocity data to properly phase the spectroscopic and photometric data before solving Eq. (2).

Welch (1994) demonstrated the advantages of using the near-infrared colour index $(V-K)$ together with the $K$-band magnitudes and made a first calibration of $F_{K}$ versus $(V-K)$ 


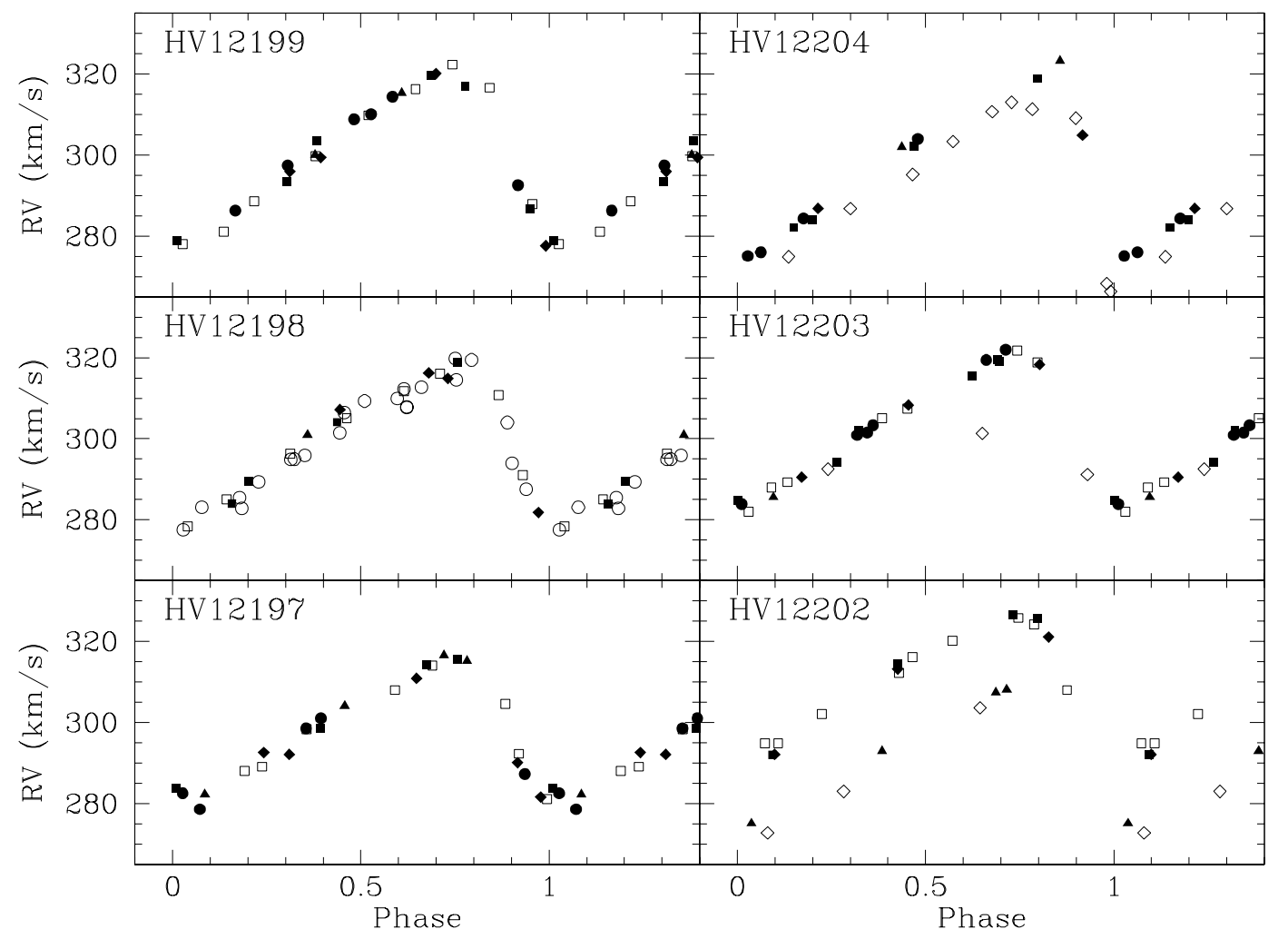

Fig. 7. The radial velocity curves for six NGC 1866 Cepheids. Our new data are marked with filled symbols, while data from the literature have been over-plotted as open symbols. The data are marked in the following way: LCO 1995: filled squares; LCO 1996: filled diamonds; LCO 2000: filled circles; CTIO: filled triangles; Storm et al. (2004a): open circles; Welch et al. (1991) HJD < 2447 520: open squares; Welch et al. (1991) $2447860<$ HJD < 2447877 : open diamonds.

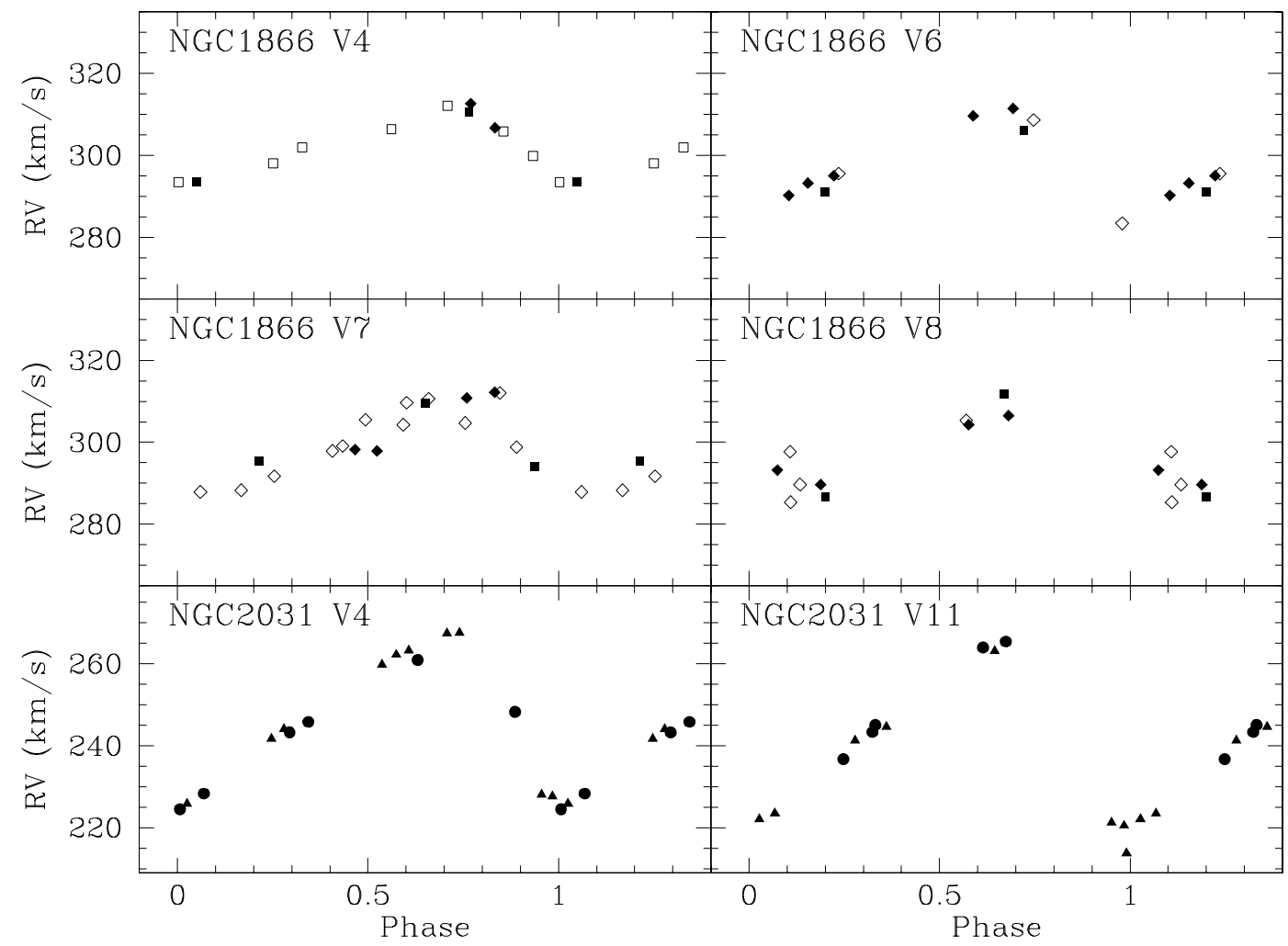

Fig. 8. The radial velocity curves for four additional NGC 1866 Cepheids and for two NGC 2031 Cepheids. The legend is as in Fig. 7. 


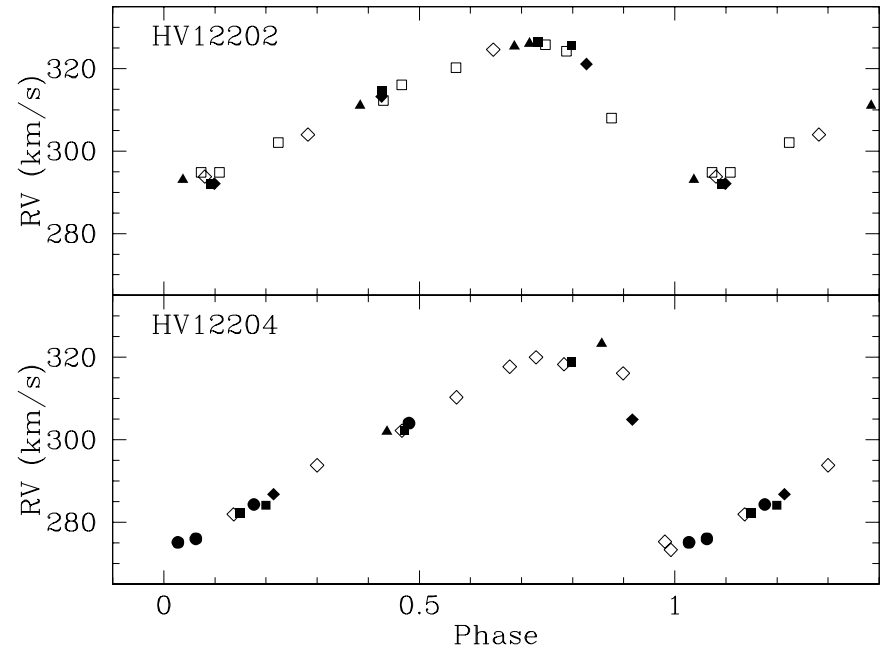

Fig. 9. The radial velocity curves for the two NGC 1866 Cepheids with suspected orbital motion after the radial velocities have been shifted as described in the text. The legend is as in Fig. 7.

Table 4. The systemic velocity, $V_{\gamma}$, computed as the phase weighted mean velocity. The random uncertainty is of the order $0.2 \mathrm{~km} \mathrm{~s}^{-1}$ and the systematic error of the order $1 \mathrm{~km} \mathrm{~s}^{-1}$.

\begin{aligned} & \hline \hline \multicolumn{1}{c}{ ID } \multicolumn{1}{c}{$\begin{array}{c}V_{\gamma} \\ {\left[\mathrm{km} \mathrm{s}^{-1}\right]}\end{array}$} \\ & \hline HV 12197 298.6 \\ & HV 12198 299.7 \\ & HV 12199 300.4 \\ & HV 12202 $303.1^{a} \\ &$ HV 12203 302.4 \\ & HV 12204 $296.5^{a} \\ &$ NGC 1866-V4 302.6 \\ & NGC 1866-V6 298.9 \\ & NGC 1866-V7 298.7 \\ & NGC 1866-V8 298.2 \\ & NGC 2031-V4 247.7 \\ & NGC 2031-V11 242.1 \\ & \hline${ }^{a}$ Stars with suspected orbital motion. \end{aligned}

on the basis of interferometric diameters of non-pulsating stars. Fouqué \& Gieren (1997) reformulated the near-infrared relation as a $F_{V},(V-K)$ relation which they calibrated, using an expanded sample of accurate interferometric measurements of non-pulsating giant stars. Later Nordgren et al. (2002) and Kervella et al. (2004a) obtained very accurate interferometric radii of Cepheids allowing them to calibrate the surface brightness method directly. For the Cepheid $\ell$ Car Kervella et al. (2004b) made a phase by phase comparison between their interferometric measurements and the values returned by the Fouqué \& Gieren relation. Recently Groenewegen (2004) has expanded and improved on the work on the non-pulsating stars. As there is excellent agreement among all these various calibrations, we choose for consistency with S04 to still adopt the
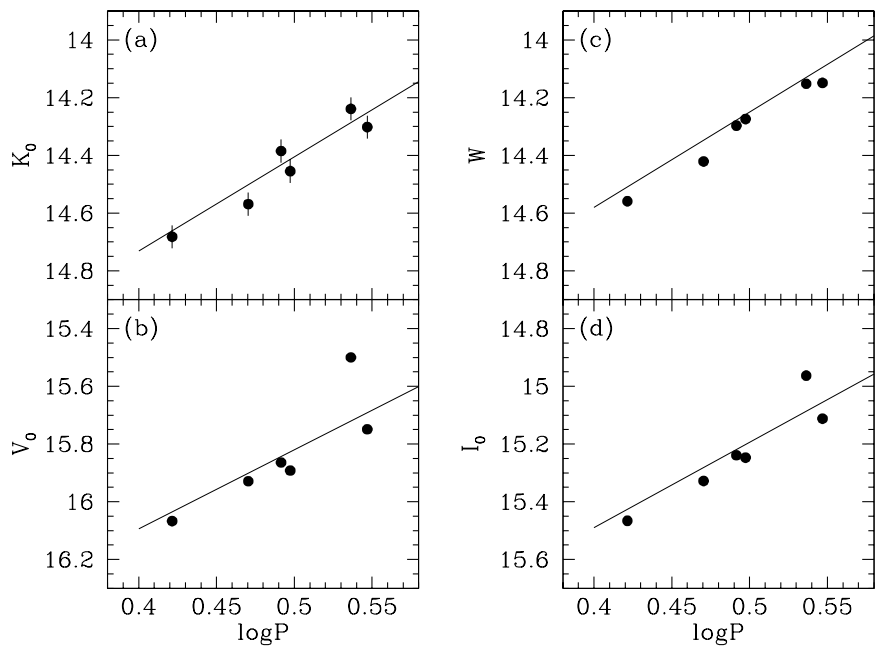

Fig. 10. Panel a) shows the observed intensity averaged $K$ magnitude vs. $\log P$ with the Persson et al. (2004) relation over-plotted. Panels b) to d) show the observed $V, I$, and $W$ magnitudes with the corresponding OGLE based relations from Fouqué et al. (2003) over-plotted.

relationship from Fouqué \& Gieren (1997). In this way the left hand side of Eq. (2) seems well established.

The right hand side of the equation contains the radius variation which, apart from the direct observables, depends through Eq. (3) on one non-observable, namely the projection factor $p$. As an error in $p$ carries directly over in the derived distance, it is of fundamental importance that $p$ is well understood. S04 and references therein argue for their choice of a weakly period dependent $p$, namely $p=1.39-0.03 \log P$ where $P$ is the pulsation period in days.

\subsection{Results}

Before applying the ISB method to the observational data we need to adopt reddenings for the individual stars. The distances derived from the ISB method are themselves very robust to errors in the assumed reddening, but the absolute magnitudes of course reflect directly any errors so introduced.

We have adopted the canonical value of $E(B-V)=0.06$ which was originally determined by van den Bergh \& Hagen (1968) from integrated $U B V$ photometry, and by Walker (1974) from stellar $U B V$ photometry. We assume an uncertainty of about $0.01 \mathrm{mag}$ on this result.

In Fig. 10 we have plotted the P-L relations in $V, I, W$, and $K$ using the adopted reddening of $E(B-V)=0.06$. Overplotted the data are the observed P-L relations based on the OGLE data (Udalski et al. 1999) as determined by Fouqué et al. (2003) based on an average LMC reddening of $E(B-V)=$ 0.10 mag. The estimated relative random errors on the mean magnitudes in the optical bands are 0.005 mag and thus smaller than the points in the diagram. In the $K$ band where the zeropoints are determined individually for each star the estimated random errors are somewhat larger, of the order 0.04 mag.

Groenewegen \& Salaris (2003) have recently claimed a reddening of $E(B-V)=0.12$ towards NGC 1866, but we do not see any support for this value in our own observational data. 
Table 5. The measured distance moduli, radii, and absolute intensity averaged magnitudes for the NGC 1866 Cepheids. The estimated error is just the formal error on the fit. The adopted reddening, $E(B-V)$, and the phase shift, $\Delta \phi$, applied to the radial velocity data are also given.

\begin{tabular}{crrrrrrrrrrrrr}
\hline \hline ID & $\log P$ & $(m-M)_{0}$ & $\sigma_{(m-M)}$ & $\begin{array}{r}R \\
{[\mathrm{mag}]}\end{array}$ & $\begin{array}{r}\sigma_{R} \\
{[\mathrm{mag}]}\end{array}$ & $\begin{array}{r}M_{B} \\
{\left[R_{\odot}\right]}\end{array}$ & $\begin{array}{r}M_{V} \\
{\left[R_{\odot}\right]}\end{array}$ & $\begin{array}{r}M_{I} \\
{[\mathrm{mag}]}\end{array}$ & $\begin{array}{r}M_{J} \\
{[\mathrm{mag}]}\end{array}$ & $\begin{array}{r}M_{K} \\
{[\mathrm{mag}]}\end{array}$ & $\begin{array}{r}M_{W} \\
{[\mathrm{mag}]}\end{array}$ & $\begin{array}{r}E(B-V) \\
{[\mathrm{mag}]}\end{array}$ & $\begin{array}{r}\Delta \phi \\
{[\mathrm{mag}]}\end{array}$ \\
\hline HV 12199 & 0.421469 & 18.336 & 0.094 & 25.0 & 1.1 & -1.737 & -2.269 & -2.870 & -3.075 & -3.672 & -3.777 & 0.060 & 0.025 \\
HV 12203 & 0.470427 & 18.481 & 0.092 & 28.3 & 1.2 & -1.989 & -2.552 & -3.153 & -3.562 & -3.930 & -4.060 & 0.060 & 0.050 \\
HV 12202 & 0.491519 & 18.289 & 0.072 & 28.5 & 1.0 & -1.815 & -2.425 & -3.050 & -3.553 & -3.922 & -3.992 & 0.060 & 0.025 \\
HV 12197 & 0.497456 & 18.165 & 0.058 & 25.9 & 0.7 & -1.712 & -2.273 & -2.918 & -3.338 & -3.728 & -3.891 & 0.060 & -0.020 \\
HV 12204 & 0.536402 & 18.202 & 0.044 & 28.3 & 0.6 & -2.243 & -2.702 & -3.239 & -3.626 & -3.981 & -4.050 & 0.060 & 0.010 \\
HV 12198 & 0.546887 & 18.314 & 0.028 & 29.8 & 0.4 & -1.989 & -2.565 & -3.202 & -3.675 & -4.030 & -4.165 & 0.060 & 0.015 \\
\hline
\end{tabular}

From Fig. 10 it is clear that the $V$ and $I$ data appear slightly too faint (disregarding the slightly peculiar star HV 12204) but an increase of the reddening of only 0.01 mag would make the data in all the diagrams agree very well with the OGLE based relations. Adopting an NGC 1866 reddening of $E(B-V)=0.12$ on the other hand, would cause all the data points in the reddening sensitive ( $V$ and $I$ ) diagrams to appear strongly $(0.15$ mag in $V$ ) over-luminous with respect to the OGLE based relations. We thus argue that the NGC 1866 reddening of $E(B-V)=0.06$ is in good agreement with the reddening scale adopted by Fouqué et al. (2003) which will also be used in the following. We stress the importance of using self-consistent reddenings and the superiority of reddening insensitive indices like $W$ and better still the $K$ band.

On the basis of the observational data presented in the previous sections we have applied the infrared surface brightness method, using the Fouqué \& Gieren (1997) calibration following the procedure explained in S04. The derived distance moduli and associated formal errors for the individual stars have been tabulated in Table 5 together with the derived absolute magnitudes. We have also tabulated the phase shifts, $\Delta \phi$, which have been applied to the radial velocity data to match the photometric angular diameter curve as discussed by S04.

For HV 12198 we find a distance modulus of 18.31, which is shorter by $0.11 \mathrm{mag}$ compared to the value of $18.42 \pm 0.1$ found by Gieren et al. (2000b). This is largely due to the fact that, as discussed in detail by S04, to avoid effects from possible shocks in the stellar atmosphere close to minimum radius, we only consider the phase interval between 0.0 and 0.8 here. An additional, but less important, reason is that we now use the bi-sector for fitting Eq. (2).

In Fig. 11 we show as an example the fit to the data for HV 12198 as well as the angular diameter variation as a function of phase.

In Fig. 12 we have plotted the derived absolute magnitudes in $V$ and $K$ as well as the Wesenheit index and the distance modulus as a function of $\log P$ with the Galactic relations over-plotted. It can be seen that the absolute magnitudes are in good agreement with the Galactic Cepheids, confirming the finding by S04 from SMC Cepheids that a metallicity effect on the absolute magnitude of these stars is small, if at all present. The Galactic relations are largely based on the data presented in S04 but expanded by four additional stars and some additional observational data from the literature,
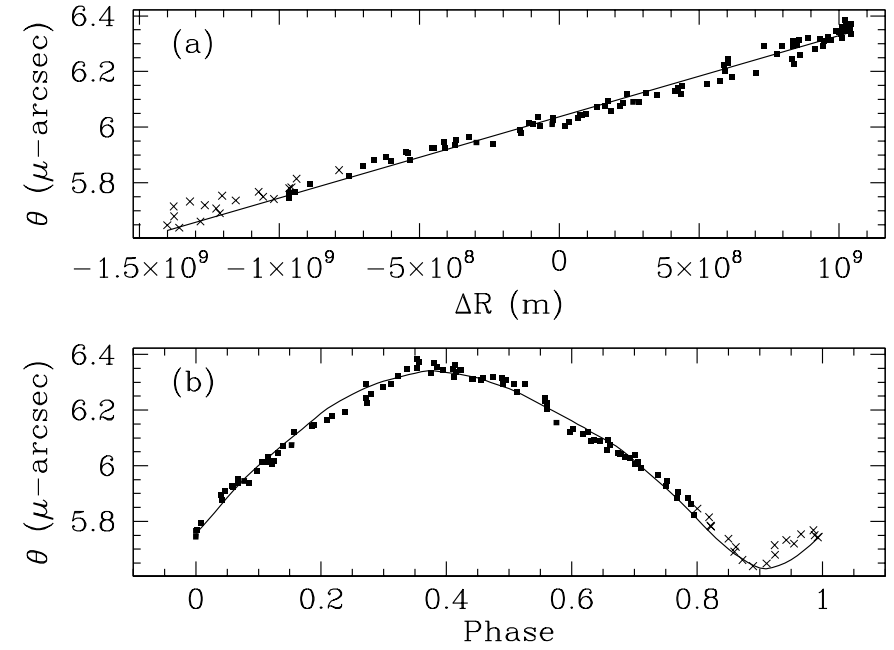

Fig. 11. Panel a) shows the observed data set of angular diameters, $\theta$ (in micro arc-seconds), versus radius variation, $\Delta R$, corresponding to Eq. (2) for the star HV 12198. The straight line is the bi-sector fit to the data in the phase interval $[0.0,0.8]$. Panel b) shows the angular diameter as a function of phase. The points correspond to the photometrically determined angular diameters and the curve corresponds to the integrated pulsational velocity curve. In both panels the crosses represent discarded points.
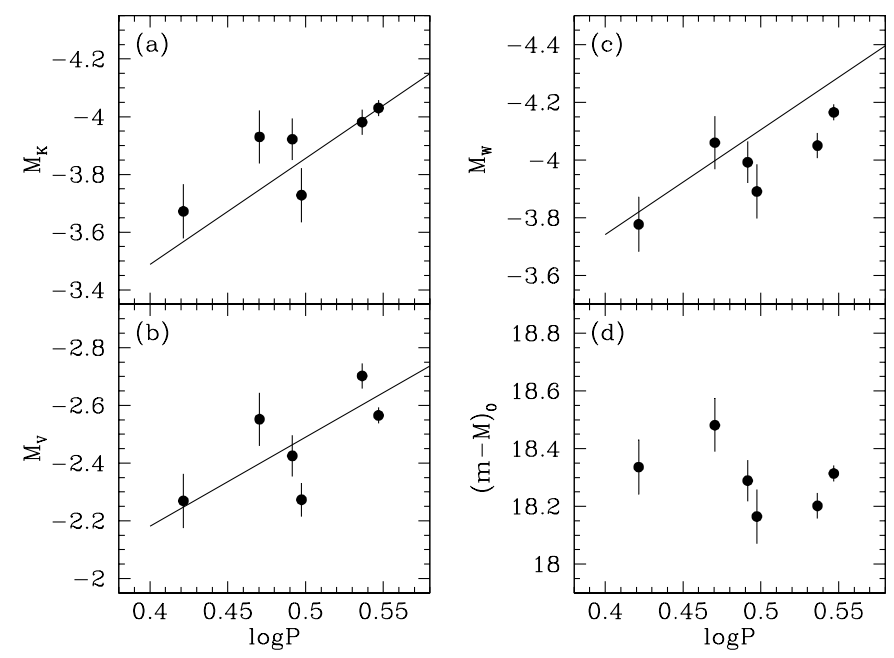

Fig. 12. Panels a) to c) show the absolute magnitudes of the Cepheids versus $\log P$ with the Galactic relation from $\mathrm{S} 04$ overplotted. Panel d) shows the derived distance moduli as a function of period. 

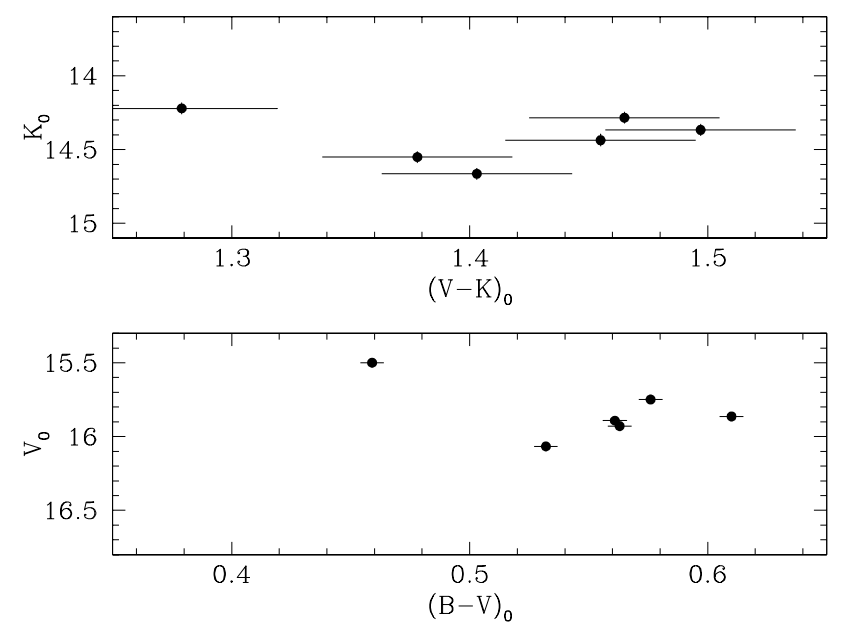

Fig. 13. The colour-magnitude diagram for the NGC 1866 Cepheids in $V,(B-V)$ and $K,(V-K)$. The bluest object is HV 12204.

see Gieren et al. (2005) for details. We note that the scatter in Fig. 12 is largely dominated by errors in the distance estimates as these correlate well with the deviations seen in the luminosities. The effect of color (temperature) on the luminosity due to the finite width of the instability strip is comparatively minor, as can be seen from Fig. 10 which is distance independent and shows a very low scatter with only HV 12204 exhibiting a significant deviation. We will later argue that this deviation can be explained by a blue companion.

\section{Discussion}

\subsection{Cluster membership of HV 12204}

Fischer et al. (1992) measured the velocity dispersion of NGC 1866 from non-variable stars to be $2.3 \mathrm{~km} \mathrm{~s}^{-1}$ and found a systemic radial velocity for the cluster of $V_{\gamma, \mathrm{NGC} 1866}=$ $301 \mathrm{~km} \mathrm{~s}^{-1}$. W91 found a systemic velocity of $V_{\gamma}=$ $293 \mathrm{~km} \mathrm{~s}^{-1}$ for HV 12204 and consequently they suspected it to be a non-member due to the inconsistent radial velocity and later works have all dismissed the star on this basis. However, with the additional radial velocity data we have presented here, it appears that HV 12204 is a single-lined spectroscopic binary exhibiting orbital motion. We do not have enough data to attempt to determine an orbit, but our data suggest that HV 12204 could very well be a member of NGC 1866. In Fig. 10 the de-reddened magnitudes of the stars have been plotted against $\log P$ with the OGLE based P-L relations from Fouqué et al. (2003) and from Persson et al. (2004) over-plotted. It is clear from these plots that the NGC 1866 Cepheids closely follow these relations. In the $V$ and $I$ bands HV 12204 appears slightly over-luminous, but the effect does not seem to be caused by a difference in distance with respect to the other NGC 1866 stars as the effect changes with wavelength and has disappeared in the $K$ band as well as in the Wesenheit index. Consequently we do not find sufficient evidence to discard HV 12204 as a possible non-member of the cluster. In Fig. 13 the colour-magnitude diagram for the NGC 1866 Cepheids has been plotted and it can be seen that HV 12204 is the bluest and brightest of the stars. We cannot exclude that the photometry is affected by an unresolved blue companion which is further supported by the fact that the $(J-K)$ color is in good agreement with that observed for the other cluster Cepheids.

\subsection{The distance to NGC 1866 and the estimated distance errors}

We now have a sample of six NGC 1866 Cepheids which we can use in the following to determine the distance to NGC 1866 and to the LMC, and to determine the size of the internal random errors of the method.

The six Cepheids are all within a radius of 3 arcmin from the cluster center and assuming conservatively a maximum cluster radius of 10 arcmin, this corresponds to a physical radius of about $150 \mathrm{pc}$ at a distance of $50 \mathrm{kpc}$. Thus the maximum error due to depth effects within NGC 1866 would be $\pm 150 \mathrm{pc}$ on a total distance of $50 \mathrm{kpc}$ which is $\pm 0.3 \%$ or $\pm 0.007 \mathrm{mag}$. For our purposes we can thus safely assume the stars to be at the same distance.

We find a weighted mean distance modulus of $18.28 \pm 0.05$ with a standard deviation of $0.11 \mathrm{mag}$. When we compare the standard deviation with the formal errors in Table 5, which range from 0.028 to 0.094 , it is obvious that the formal errors significantly (by about a factor of two) underestimate the true errors. It can also be seen from the table that the formal errors spread significantly, even though the data quality is reasonably uniform. In fact the variation of the estimated errors mostly reflect the overall agreement between the shape of the two angular diameter curves and to a much lesser extent the random noise of the data. In this way a few stars carry a possibly unrealistic high relative weight. We thus prefer to use the unweighted mean as the most unbiased estimator, leading to $(m-M)_{0}=18.30 \pm 0.05^{2}$, in apparent excellent agreement with the main-sequence fitting distance from Walker et al. (2001).

Barnes et al. (2003) have performed a very thorough statistical analysis of the optical surface brightness method using Bayesian techniques. A similar investigation of the infrared surface brightness method is currently in press (Barnes et al. 2005). Preliminary results indeed suggest that the formal errors of the type reported here are underestimates but that the derived individual distances remain unchanged.

\subsection{The LMC distance}

Even though NGC 1866 is some way away from the LMC center we can attempt, at least formally, to determine the distance to the LMC on the basis of these data.

As NGC 1866 is a young object it must be close to the LMC disk. In Fig. 10 it seems as if the NGC 1866 Cepheids are slightly (of the order $0.05 \mathrm{mag}$ ) fainter than the average LMC Cepheids in the $V$ and $I$ bands when disregarding HV 12204, but very close to the mean value in the reddening insensitive $W$ index and in the $K$ band. As previously discussed the deviation in the $V$ and $I$ bands would disappear if the reddening were increased by $\Delta E(B-V)=0.01$, well within the

2 This is our best distance estimate at present, but we caution the reader to take into account the caveats in Sect. 7.4. 
estimated uncertainty of the reddening. Groenewegen \& Salaris (2003) have suggested that NGC 1866 is more distant than the surrounding disk population. At the same time the disk model of van der Marel \& Cioni (2001) at this position in the north eastern part of the LMC (RA: $05: 13: 39$, Dec: $-65: 27.9$ $(2000.0))$ says that the disk is closer than the LMC center by 0.059 mag. These two effects largely cancel out and confirm our finding that the NGC 1866 Cepheids are close to the average LMC Cepheid distance, so we choose for the present purposes not to apply any correction for geometry, but add in quadrature an additional uncertainty of $0.05 \mathrm{mag}$. We thus formally find an LMC distance modulus of $(m-M)_{0,\{\mathrm{LMC}}=$ $18.30 \pm 0.07$ mag. We note that the derived modulus is on the short side of the available estimates, but as the following discussion will show, this should only be considered a preliminary estimate of the LMC distance modulus.

\subsection{Comparing the Period-Luminosity relations}

The absolute magnitudes which we have derived for the LMC Cepheids appear in excellent agreement with the values expected from the Galactic relations derived using exactly the same precepts by S04. In Figs. 14-16 we have overplotted these stars on the Galactic Cepheids. We have also overplotted the SMC Cepheids from S04, which have a metallicity of $[\mathrm{Fe} / \mathrm{H}]=-0.7$ which is not very different from the metallicity of the NGC 1866 Cepheids of $[\mathrm{Fe} / \mathrm{H}]=-0.5$ from Hill et al. (2000). The filled lines in these plots represents the linear Galactic relations fit to the Galactic Cepheids. It is clear that the low metallicity stars follow this relation quite well and there is no obvious sign of a difference in slope between the low metallicity and high metallicity populations. From the perspective of the ISB method the two populations appear to follow similar $\mathrm{P}-\mathrm{L}$ relations.

However, the dashed lines overplotted in Figs. 14-16 represent the observed apparent $\mathrm{P}-\mathrm{L}$ relations shifted to the formal LMC distance of $(m-M)_{0}=18.30$ mag. As already noted and discussed by Fouqué et al. (2003), Tammann et al. (2003), and S04 these slopes differ from the slopes found from the Galactic sample as determined from the ISB method. Now we can see indications that these slopes also might differ from the slopes determined directly for the low metallicity stars from the ISB method. This suggests that there could be a problem either with the slopes of the apparent P-L relations, which is highly unlikely, or with the slopes derived from the ISB method. To clarify this point it will be necessary to study a significantly larger sample of LMC Cepheids spanning a large range of periods. Gieren et al. (2005) will present the first results from such an expanded sample.

If we can indeed confirm the suggestion that the LMC and SMC Cepheids follow the same P-L relation from the ISB method as the Cepheids in the Galaxy, this will mean that the P-L relation is afterall a good distance indicator as the slope is not dependent on metallicity. However, the ISB method itself will then need mending and the distances and absolute magnitudes derived here and in S04 will need revision.

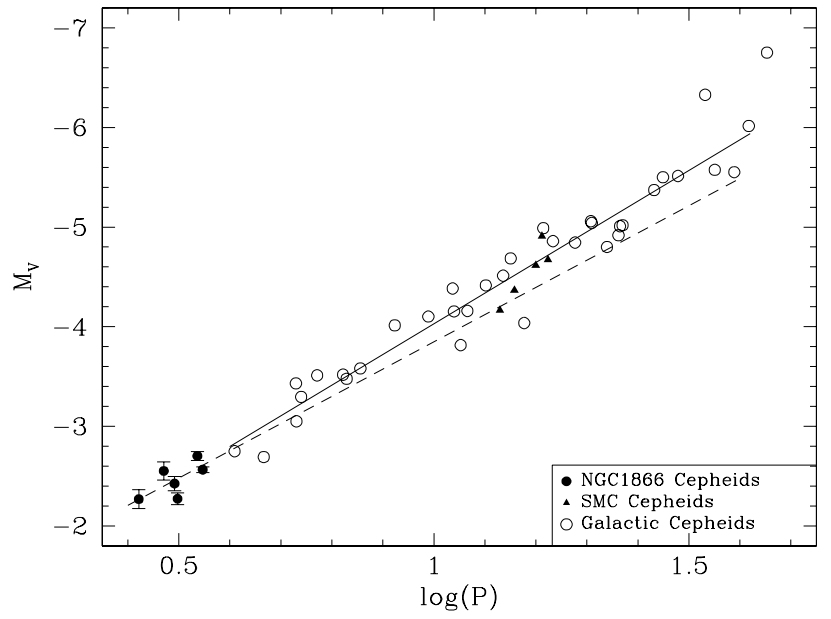

Fig. 14. The P-L relation for Galactic Cepheids with the NGC 1866 Cepheids overplotted. The dashed line is the OGLE P-L relation for an adopted LMC distance of $(m-M)_{0}=18.30 \mathrm{mag}$.

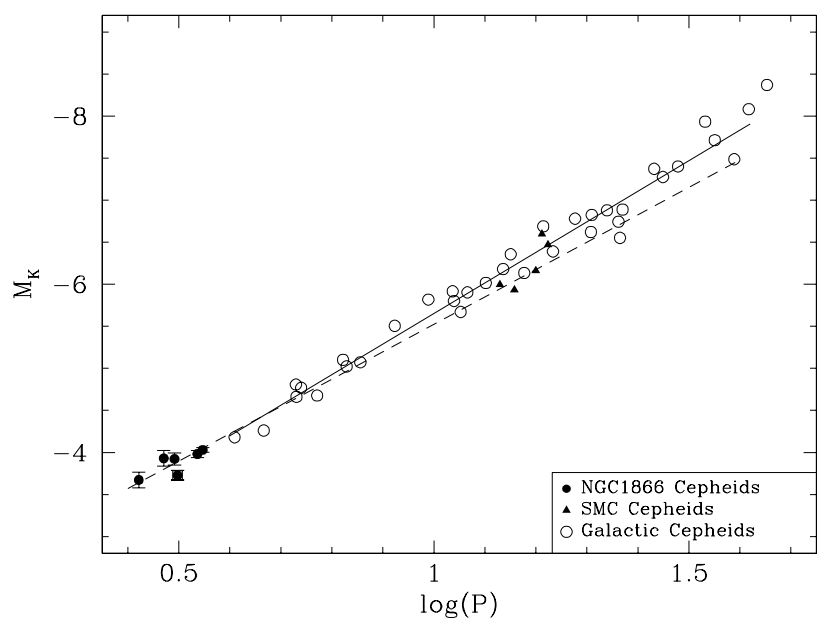

Fig. 15. The P-L relation for Galactic Cepheids with the NGC 1866 Cepheids overplotted. The dashed line is the Persson et al. (2004) $\mathrm{P}-\mathrm{L}$ relation for an adopted LMC distance of $(m-M)_{0}=18.30 \mathrm{mag}$.

It is relevant to note that the theoretical models of Caputo et al. (2000) predict that the metallicity effect on the luminosity for short period Cepheids is very small, exactly as seen in Fig. 14. The predictions for the longer period stars, and thus for the slope, are tested with the expanded sample of Gieren et al. (2005)

\section{Conclusions}

We have presented new near-IR light curves for six NGC 1866 Cepheids and high precision radial velocity curves for six NGC 1866 Cepheids and two Cepheids in NGC 2031.

Using these data we have shown that HV 12204 is most likely a member of NGC 1866 but subject to orbital motion, just like HV 12202.

Following the precepts in S04 we have derived absolute magnitudes and distances to the six NGC 1866 Cepheids. The distances exhibit a standard deviation of 0.11 mag suggesting 


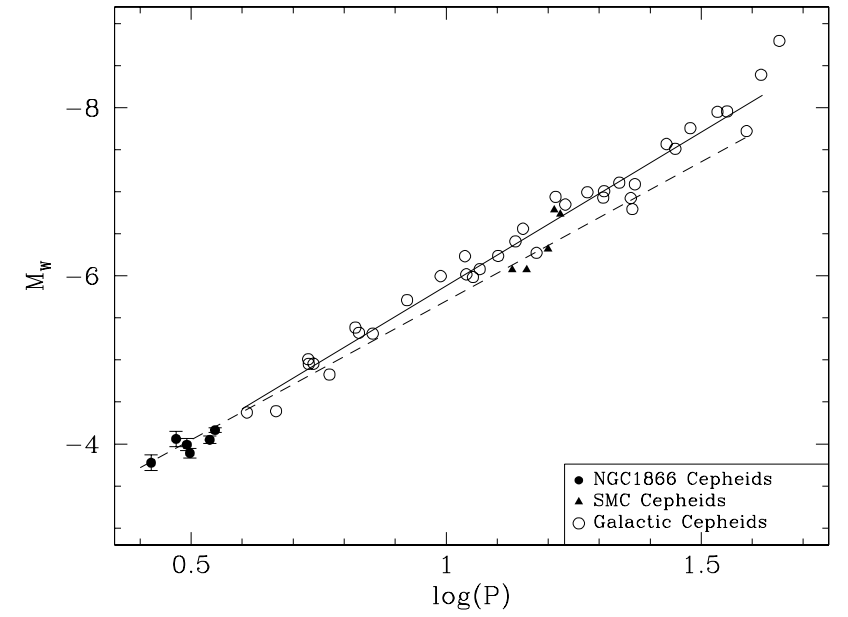

Fig. 16. The P-L relation for Galactic Cepheids with the NGC 1866 Cepheids overplotted. The dashed line is the OGLE P-L relation for an adopted LMC distance of $(m-M)_{0}=18.30 \mathrm{mag}$.

that the formal error estimates on the individual distance moduli are underestimated by about a factor of two.

Formally we find a distance modulus of $(m-M)_{0}=$ $18.30 \pm 0.05$ to NGC 1866 , but due to the potential problems with the method in its present form uncovered here, this should be regarded as a preliminary estimate. Similarly the formal LMC modulus of $(m-M)_{0}=18.30 \pm 0.07$ might need revision.

The absolute magnitudes $M_{V}, M_{K}$ and the Wesenheit in$\operatorname{dex} M_{W}$ for the NGC 1866 Cepheids are all in excellent agreement with the P-L relations determined by S04 for Galactic Cepheids.

The NGC 1866 Cepheids with a metallicity of $[\mathrm{Fe} / \mathrm{H}]=$ -0.5 and the SMC Cepheids from S04 with a metallicity of $[\mathrm{Fe} / \mathrm{H}]=-0.7$ appear to follow largely the same P-L relation as the Galactic Cepheids, in particular they seem to follow a similar slope. However, this slope is different from the slope of the directly observed apparent P-L relations in $K, V$, and $W$ suggesting an intrinsic systematic error with the ISB method which casts doubt on the distances and absolute magnitudes derived by the method. On the other hand if this problem originates with the ISB method itself, this might resolve the very unpleasant problem of the slope of the P-L relation depending on metallicity.

Acknowledgements. We thank Roeland van der Marel for providing his code for computing the LMC distance correction for the stars. This work has benefitted from the use of the McMaster Cepheid database (http://dogwood.physics.mcmaster.ca/Cepheid/) maintained by D.L. Welch. WG gratefully acknowledges financial support from the Chilean Center for Astrophysics FONDAP 15010003. OSIRIS is a collaborative project between the Ohio State University and Cerro Tololo Inter-American Observatory (CTIO) and was developed through NSF grants AST 90-16112 and AST 92-18449.

\section{References}

Arp, H., \& Thackeray, A. D. 1967, ApJ, 149, 73

Barnes, T. G., \& Evans, D. S. 1976, MNRAS, 174, 489

Barnes, T. G., Evans, D. S., \& Parsons, S. B. 1976, MNRAS, 174, 503
Barnes, T. G., Dominy, J. F., Evans, D. S., et al. 1977, MNRAS, 178, 661

Barnes, T. G., Jefferys, W. H., Berger, J. O., et al. 2003, ApJ, 592, 539

Barnes, T. G., Storm, J., Jefferys, W. H., Gieren, W. P., \& Fouqué, P. 2005, ApJ, in press [arXiv:astro-ph/0506077]

Benedict, G. F., McArthur, B. E., Fredrick, L. W., et al. 2002, AJ, 123 , 473

Bertelli, G., Bressan, A., Chiosi, C., Mateo, M., \& Wood, P. R. 1993 , ApJ, 412, 160

Brocato, E., Buonanno, R., Castellani, V., \& Walker, A. R. 1989, ApJS, 71, 25

Brocato, E., Castellani, V., Di Carlo, E., Raimondo, G., \& Walker, A. R. 2003, AJ, 125, 3111

Brocato, E., Caputo, F., Castellani, V., Marconi, M., \& Musella, I. 2004, AJ, 128, 1597

Caputo, F., Marconi, M., \& Musella, I. 2000, A\&A, 354, 610

Côté, P., Welch, D. L., Mateo, M., Fischer, P., \& Madore, B. F. 1991, AJ, 101, 1681

Fouqué, P., \& Gieren, W. P. 1997, A\&A, 320, 799

Fouqué, P., Storm, J., \& Gieren, W. P. 2003, in Stellar Candles, ed. D. Alloin, \& W. Gieren, Lect. Notes Phys. (Springer Verlag), 635, 21

Fischer, P., Welch, D. L., Côté, P., Mateo, M., \& Madore, B. F. 1992, AJ, 103, 857

Freedman, W. L., Madore, B. F., Gibson, B. K., et al. 2001, ApJ, 553, 47

Gibson, B. K. 2000, Mem. Soc. Astron. Ital., 71, 693

Gieren, W. P., Gómez, M., Storm, J., et al. 2000a, ApJS, 129, 111

Gieren, W. P., Storm, J., Fouqué, P., Mennickent, R., \& Gómez, M. 2000b, ApJ, 533, L107

Gieren, W. P., Storm, J., Barnes, T. G., et al. 2005, ApJ, 627, 224

Groenewegen, M. A. T., \& Salaris, M. 2003, A\&A, 410, 887

Groenewegen, M. A. T. 2004, MNRAS, 353, 903

Hill, V., Francois, P., Spite, M., Primas, F., \& Spite, F. 2000, A\&A, 364, L19

Hilker, M., Richtler, T., \& Gieren, W. P. 1995, A\&A, 294, 648

Kervella, P., Bersier, D., Mourard, D., et al. 2004a, A\&A, 423, 327

Kervella, P., Fouqué, P., Storm, J., et al. 2004b, ApJ, 604, L113

Kurtz, M. J., \& Mink, D. J. 1998, PASP, 110, 934

Mateo, M. 1992, PASP, 104, 824

Nordgren, T. E., Lane, B. F., Hindsley, R. B., \& Kervella, P. 2002, ApJ, 123, 3380

Persson, S. E., Murphy, D. C., Krzeminski, W., Roth, M., \& Rieke, M. J. 1998, AJ, 116, 2475

Persson, S. E., Madore, B. F., Krzemiński, W., et al. 2004, AJ, 128, 2239

Shapley, H., \& Nail, V. M. 1950, AJ, 55, 249

Schecter, P. L., Mateo, M., \& Saha, A. 1993, PASP, 105, 1342

Storm, J., Andersen, J., Blecha, A., \& Walker, M. F. 1988, A\&A, 190, L18

Storm, J., Carney, B. W., Gieren, W. P., et al. 2004a, A\&A, 415, 521

Storm, J., Carney, B. W., Gieren, W. P., et al. 2004b, A\&A, 415, 531 (S04)

van den Bergh, S., \& Hagen, G. L. 1968, ApJ, 73, 569

Tammann, G. A., Sandage, A., \& Reindl, B. 2003, A\&A, 404, 423

Tonry, J. L., \& Davis, M. 1979, AJ, 84, 1511

Udalski, A., Soszyński, I., Szymański, M., et al. 1999, Acta Astron., 49,223

van der Marel, R. P., \& Cioni, M.-R. L. 2001, AJ, 122, 1807

Walker, A. R. 1987, MNRAS, 225, 627

Walker, M. F. 1974, MNRAS, 169, 199

Walker, A. R., Raimondo, G., Di Carlo, E., et al. 2001, ApJ, 560, L139

Welch, D. L., Côté, P., Fischer, P., Mateo, M., \& Madore, B. F. 1991, AJ, 101, 490 (W91)

Welch, D. L., \& Stetson, P. B. 1993, AJ, 105, 1813

Welch, D. L. 1994, AJ, 108, 1421 


\section{Online Material}




\section{Appendix A: Photometric transformations}

\section{A.1. Transformation to the common instrument system}

The $J K$ data from all the observing runs were transformed to the Las Campanas 1998 instrument system before being transformed to the standard system.

\section{A.1.1. The Las Campanas and CTIO data}

In the case of the Las Campanas observing runs and the CTIO run, the colour terms for the transformations to the Las Campanas instrument system were determined using a data set for the LMC cluster NGC 2136. This field contains a large number of stars without becoming seriously crowded. The central part containing the cluster itself was disregarded to reduce the number of stars with systematic errors due to poor sky subtraction or neighbour subtraction. For each set of $J$ and $K$ exposures the transformation to the Las Campanas (LCO) instrument system was determined, assuming relations of the form

$$
K_{\mathrm{LCOinst}}=K_{\mathrm{inst}}+\alpha_{K}(J-K)_{\mathrm{inst}}+\beta_{K}
$$$$
J_{\text {LCOinst }}=J_{\text {inst }}+\alpha_{J}(J-K)_{\text {inst }}+\beta_{J} \text {. }
$$

For each observing run the resulting slopes $\left(\alpha_{J}\right.$ and $\left.\alpha_{K}\right)$ were averaged. The Las Campanas data from the other observing runs did, as expected, not exhibit significant colour terms, and we have assumed a value of zero for $\alpha$ in the actual transformations. For the CTIO data there were slight colour terms of $\alpha_{K}=-0.03 \pm 0.01$ and $\alpha_{J}=+0.03 \pm 0.01$.

The individual $J$ and $K$ data sets were then reanalyzed with the slopes fixed at the adopted values leaving only the zero point offsets $\beta_{J}$ and $\beta_{K}$ to be fitted. Only stars with estimated errors less than $0.05 \mathrm{mag}$ were used in this fit and the known variables were of course excluded.

\section{A.1.2. The ESO data}

NGC 2136 was not observed during the ESO observing runs. Instead we observed 11 standard stars from the list of Carter \& Meadows (1995), during the night Dec. 21, 1996, to determine the colour terms. The stars span a colour range from $(J-K)=$ -0.02 to 0.96 . For the $K$-band we found no significant colour term and in the $J$-band we found $\alpha_{J}=+0.06 \pm 0.02$. This is in good agreement with the value quoted in the user manual for this colour range. As the colour range for our science targets as well as for the comparison stars in these fields are all very similar $( \pm 0.2 \mathrm{mag})$, the exact value of the colour term is not very important, as long as it is small.

Carter (1993) gives the transformation for normal stars to the CIT/CTIO system as

$$
\begin{aligned}
K_{\mathrm{CIT}} & =K_{\mathrm{C}}-0.021(J-K)_{\mathrm{C}}-0.002 \\
(J-K)_{\mathrm{CIT}} & =(J-K)_{\mathrm{C}}-0.105(J-K)_{\mathrm{C}}-0.001
\end{aligned}
$$

with standard errors of $0.01 \mathrm{mag}$. Inserting the previously found colour terms for the ESO data in these equations gives:

$$
(J-K)_{\mathrm{C}}=(J-K)_{\mathrm{ESO}}+0.06(J-K)_{\mathrm{ESO}}
$$

$$
\begin{aligned}
& =1.06(J-K)_{\mathrm{ESO}} \\
(J-K)_{\mathrm{CIT}} & =(J-K)_{\mathrm{C}}-0.105(J-K)_{\mathrm{C}}-0.001 \\
& \approx 0.895(J-K)_{\mathrm{C}} \\
& =0.895 \times 1.06(J-K)_{\mathrm{ESO}} \\
& =0.949(J-K)_{\mathrm{ESO}} .
\end{aligned}
$$

This is very similar to the colour equation given by Persson et al. (1998) (hereinafter P98) $(J-K)_{\mathrm{CIT}}=0.954(J-K)_{\mathrm{LCO}}$ between the CIT and LCO systems. As the slopes of these two relations are almost identical and the colour range is very small ( $\approx 0.4 \mathrm{mag}$ ) we conclude that we only need to apply a simple offset to the ESO data to bring the dataset onto the LCO instrument system.

\section{A.2. Transformation to the CIT standard system}

On the nights Dec. 27 and 28, 1998 at LCO we observed eight different standard stars from the list of P98 with a small range in colour $(0.24<(J-K)<0.37)$. The stars were observed several times during the nights.

We have performed synthetic aperture photometry on these stars using the photcal package within IRAF and we determine the zero point shifts $Z_{J}$ and $Z_{K}$ to the LCO standard system (P98) on the basis of the following relations:

$J_{\mathrm{LCO}}=J_{\text {LCOinst }}+k_{J} X+Z_{J}$

$K_{\mathrm{LCO}}=K_{\mathrm{LCOinst}}+k_{K} X+Z_{K}$

$X$ indicates the airmass. We have adopted the canonical airmass terms of $k_{J}=0.10$ and $k_{K}=0.08$ as quoted by P98 as our data did not suggest a significantly different value.

Similarly, synthetic aperture photometry was performed on isolated stars in the science frames. Growth curves were fitted for both the science and standard star data. On the basis of these data a number of reference stars in each field could be calibrated. The remaining time series photometry, which was already transformed to the LCO instrument system as described above, could then be offset to match these reference stars using the transformations from Eqs. (A.11)-(A.12).

The resulting light curves were averaged for each dithered observation set consisting of typically 5 or 6 exposures. The averaged magnitudes were finally transformed to the CIT system using the transformation given by P98:

$$
\begin{aligned}
K_{\mathrm{CIT}} & =K_{\mathrm{LCO}} \\
(J-K)_{\mathrm{CIT}} & =0.954(J-K)_{\mathrm{LCO}}+0.015 .
\end{aligned}
$$

The $K$ and $(J-K)$ photometric measurements are tabulated in Table 1 and the $K$ and $(J-K)$ light curves are plotted together with the $(V-K)$ colour curve in Figs. 1-6.

The $(V-K)$ colour was derived by combining the $V$ data from Gieren et al. (2000a) with the $K$-band data presented here. As the data were obtained at different times it was necessary to first smooth the $K$-band data and then interpolate in phase to derive a $(V-K)$ colour corresponding to each $V$ observation. The smoothing was done by computing the weighted mean of 5 points in phase and magnitude and interpolating linearly 
between the resulting points. This approach results in fewer artifacts than a Fourier fit and thus gives a better representation of the data. Due to the low amplitude of the $K$-band light curve any remaining inaccuracies in the smoothed $K$-band light curve causes only slight changes in the resulting $(V-K)$ curve.

The photometric errors as returned by DoPHOT are tabulated with the magnitudes in Table 1. Due to the fact that the stars have been observed with individual pointings as they did not fit into the limited field of view of the near-IR cameras, the zero points for each field have been determined independently using non-variable stars within the fields. We estimate that the uncertainty on these zero points is $0.04 \mathrm{mag}$. 